\title{
Initial stages of chain formation in a single layer of (In,Ga)As quantum dots grown on GaAs (100)
}

\author{
M. Schmidbauer ${ }^{\mathrm{a})}$ \\ Institut für Kristallzüchtung, Max-Born-Straße 2, D-12489 Berlin, Germany \\ Zh. M. Wang, Yu. I. Mazur, ${ }^{\text {b) }}$ P. M. Lytvyn, ${ }^{\text {c) }}$ and G. J. Salamo \\ Department of Physics, University of Arkansas, Fayetteville, Arkansas 72701 \\ D. Grigoriev, ${ }^{\text {d) }}$ P. Schäfer, and R. Köhler \\ Institut für Physik, Humboldt-Universität zu Berlin, Newtonstraße 15, D-12489 Berlin, Germany \\ M. Hanke \\ Institut für Physik, Martin-Luther-Universität Halle-Wittenberg, Hoher Weg 8, D-06120 Halle/Saale, \\ Germany
}

(Received 27 March 2007; accepted 28 May 2007; published online 28 August 2007)

\begin{abstract}
The self-organized formation of $\operatorname{In}_{0.40} \mathrm{Ga}_{0.60}$ As quantum dot chains was investigated using x-ray scattering. Two samples were compared grown on $\operatorname{GaAs}(100)$ by molecular beam epitaxy. The first sample with a single layer of $\mathrm{In}_{0.40} \mathrm{Ga}_{0.60}$ As dots shows weak quantum dot alignment and a corresponding elongated shape along [01ㅣ], while the top layer of a multilayered $\mathrm{In}_{0.40} \mathrm{Ga}_{0.60} \mathrm{As} / \mathrm{GaAs}$ sample exhibits extended and highly regular quantum dot chains oriented along [0 $\overline{1} 1]$. Numerical calculations of the three-dimensional strain fields are used to explain the initial stages of chain formation by anisotropic strain relaxation induced by the elongated dot shape. (0) 2007 American Institute of Physics. [DOI: 10.1063/1.2775801]
\end{abstract}

In the Stranski-Krastanow growth mode, the growth conditions can be optimized to produce semiconductor nanostructures of nearly identical size and shape. However, often only a random spatial distribution is observed for a single layer of quantum dots (QDs). ${ }^{1}$ This is caused by kinetic limitations which are particularly relevant at high growth rates and low growth temperatures. On the other hand, growth at elevated temperature may lead to ordering phenomena. As an example, extended chains of QDs arranged along the [0 $\overline{1} 1]$ direction have been observed in the growth of multiple layers of (In,Ga)As on GaAs (100). ${ }^{2,3}$ The formation mechanism of these (In,Ga)As QD chains-in particular the initial stages of ordering - is not fully understood yet.

Growth at high temperatures and at low growth rates ensures conditions close to thermodynamical equilibrium. This reinforces the role of energy minimization and thus the importance of lattice strains in the evolution of QD ordering. Surface diffusion and strain relaxation are often correlated: For example, adatom diffusion on a $\operatorname{GaAs}(100)$ surface shows a pronounced anisotropy which is caused by the $(2$ $\times 4)$ surface reconstruction. In particular, the adatom diffusion along $[0 \overline{1} 1]$ is larger than that along [011]. This leads to greater strain relaxation along $[0 \overline{1} 1]$ which is-during further multilayer growth-transferred to succeeding layers, eventually leading to an asymmetric separation between neighboring dots. The evolution of highly ordered QD chains could thus be a result of both anisotropic surface diffusion

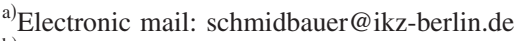

${ }^{\text {b) }}$ Electronic mail: ymazur@uark.edu

${ }^{c)}$ On leave from: Institute of Semiconductor Physics, National Academy of Sciences, Prospect Nauki 45, 03028 Kiev, Ukraine.

${ }^{d)}$ Current address: Fakultät für Physik, Universität Karlsruhe (TH), Postfach 6980, D-76128 Karlsruhe, Germany.
}

and anisotropic vertical strain inheritance in the multiple layers of QDs. ${ }^{4,5}$

In this letter, we will demonstrate that the anisotropic strain field of a QD which is elongated along [011] plays an essential role in the formation of QD chains in multilayered samples. We will focus on two samples grown under identical conditions. Sample 1 consists of a single $\operatorname{In}_{0.40} \mathrm{Ga}_{0.60} \mathrm{As}$ QD layer, while sample 2 represents a stack of 16.5 periods of $\mathrm{In}_{0.40} \mathrm{Ga}_{0.60}$ As QDs embedded in 120 ML thick GaAs spacer layers with the top layer consisting of $\operatorname{In}_{0.40} \mathrm{Ga}_{0.60} \mathrm{As}$ QDs. Both samples were grown by solid-source molecular beam epitaxy on GaAs(100) substrates. The growth conditions are described in detail elsewhere. ${ }^{3,4,6}$ Briefly, after desorption of the native oxide at $580{ }^{\circ} \mathrm{C}$, a half micrometer thick GaAs buffer layer was grown at $600{ }^{\circ} \mathrm{C}$. For the deposition of the $\operatorname{In}_{0.40} \mathrm{Ga}_{0.60}$ As QD structure $\left(0.36 \mathrm{ML} \mathrm{s}^{-1}\right)$, the substrate temperature was lowered to $540{ }^{\circ} \mathrm{C}$. For sample 2, the GaAs capping of the QDs was performed with the first 60 $\mathrm{ML}$ at $540{ }^{\circ} \mathrm{C}$ and the last $60 \mathrm{ML}$ at $600{ }^{\circ} \mathrm{C}$.

The atomic force micrograph (AFM) of sample 1 [Fig. 1(a)] shows that the QDs lign up into short chains that are directed along [011]. Moreover, short fragments of QD chains oriented along the planar $\langle 001\rangle$ directions are also observed. By contrast, the top layer of the multilayered stack of (In, Ga)As QDs consists of extended QD chains directed along [0 $\overline{1} 1]$ [Fig. 1(b)]. These chains contain monodisperse islands, they are highly regular, and their mutual spacings along [011] are well defined. A quantitative analysis of both AFM images [Figs. 1(a) and 1(b)] reveals that the dots exhibit an approximate height of $h=7 \mathrm{~nm}$. However, while the multilayered sample contains rotationally symmetric dots with a base diameter of about $w=60 \mathrm{~nm}$, the dots of the single layered sample are slightly elongated along $[0 \overline{1} 1]$ with approximate bases of $w_{[011]}=60 \mathrm{~nm}$ and $w_{[011]}=40 \mathrm{~nm}$. 

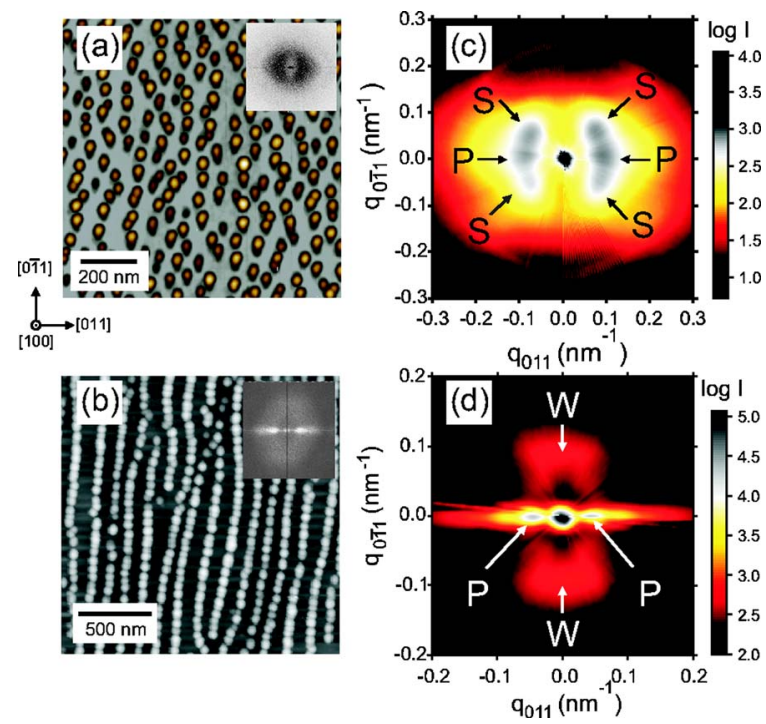

FIG. 1. (Color online) Atomic force micrographs of (a) a single layer of $\mathrm{In}_{0.40} \mathrm{Ga}_{0.60} \mathrm{As}$ QDs grown on GaAs(100) (sample 1) and of (b) a 16.5 period multilayer of $\operatorname{In}_{0.40} \mathrm{Ga}_{0.60} \mathrm{As} / \mathrm{GaAs} \mathrm{QDs}$ grown on GaAs(100) (sample 2). (c) and (d) are corresponding GISAXS intensity patterns on a logarithmic color scale. The insets in (a) and (b) are fast Fourier transforms of the corresponding AFM image.

QDs with an elongated base have been the subject of extensive discussion in the literature owing to the induced anisotropic optical properties. ${ }^{7,8}$ Since real surfaces are often strongly anisotropic, significant deviations from highly symmetrical nanoscale objects (e.g., hemispherical domes ${ }^{9}$ or lens-shaped rotationally symmetric islands ${ }^{10}$ ) were frequently observed. This anisotropy in surface-free energy may cause faceting of the island surface, resulting in a complicated final island shape. The situation can be further complicated by kinetic limitations during growth in that anisotropic surface diffusion lengths may also have a large impact on the QD shape. ${ }^{11}$ Consequently, a large variety of quantum dot shapes has been suggested (e.g., Refs. 12 and 13) and reported experimentally (e.g., Refs. 7 and 14-17).

Grazing-incidence small-angle $x$-ray scattering (GISAXS) and grazing incidence $x$-ray diffraction (GIXD) have been performed at an x-ray wavelength of $\lambda=1.55 \AA$. In order to ensure maximum sensitivity to the QD top layer, the angle of incidence was chosen slightly below the critical angle of total external reflection $\left(\alpha_{c}=0.31^{\circ}\right)$. The experiments were carried out at the synchrotron radiation facilities in Hamburg, Germany (HASYLAB, beamline BW2) and Grenoble, France (ESRF, beamline ID10B).

The in-plane GISAXS intensity pattern for the multilayered sample [Fig. 1(d)] shows pronounced features which are related to the lateral ordering of the QDs. Beside the strong specularly reflected beam at $q=0$ very sharp satellite peaks $(P)$ are observed in the [011] direction. These are related to the strong chain-chain positional correlation, and from the peak positions we can evaluate a mean chain-chain distance of $d=130 \mathrm{~nm}$ which is in good agreement with the corresponding AFM image shown in Fig. 1(b). From the peak width $\delta q_{011}=0.013 \mathrm{~nm}^{-1}$ of $P$, we can evaluate a lateral correlation length of about $L_{011}=2 \pi / \delta q_{011}=480 \mathrm{~nm}$; i.e., the ordering extends over more than four chains. The positional correlation of the QDs within the chains-i.e., along
$W$ [Fig. 1(d)] with a corresponding lateral correlation length of about $L_{011}=80 \mathrm{~nm}$, which is fairly small.

The in-plane GISXAS intensity pattern of the single layer of QDs [Fig. 1(c)] looks quite different as compared to that of the multilayered sample. We again observe two prominent peaks $(P)$ in the direction along [011] separated by $\Delta q_{011}=0.19 \mathrm{~nm}^{-1}$ which correspond to a spacing of $d$ $=65 \mathrm{~nm}$ in real space. A comparison with the AFM image in Fig. 1(a) suggests that the peaks $P$ are created by positional correlation between neighboring chains. Evaluation of the GISAXS peak width $\delta q_{011}=0.1 \mathrm{~nm}^{-1}$ of $P$ in the direction along [011] leads to a lateral correlation length of $L_{011}$ $=2 \pi / \delta q_{011}=70 \mathrm{~nm}$, indicating short range ordering between two neighboring QD chains. On the other hand, the $\mathrm{x}$-ray diffuse scattering does not show corresponding peaks in the direction along $[0 \overline{1} 1]$. This proves that the chains are aligned along [011] but the QDs are not remarkably ordered within these chains. Thus, although less pronounced, the ordering of the QD chains within a single layer is qualitatively similar to that of a multiple layer of QDs.

Four additional weak peaks $(S)$ are observed for the diffuse scattering from the single layer [Fig. 1(c)]. Their positions suggest additional QD alignment along the two planar $\langle 001\rangle$ directions which are the elastically soft directions of a group III-V semiconductor crystal with zinc-blende structure. In these directions, short fragmented QD chains are observed [Fig. 1(a)].

Superimposed on the peaks $S$ and $P$ we find in Fig. 1(c) a weak elliptically shaped intensity distribution with the long axis directed along [011]. This pattern is a direct fingerprint of the elongated shape of the individual QDs along [011]. From the lateral aspect ratio of the intensity distribution in the two planar directions [011] and [011], the aspect ratio $\alpha$ of the lateral QD extensions can be estimated as $\alpha$ $=w_{011} / w_{011} \approx 1.5$. This can be also inspected in the corresponding fast Fourier transform of the AFM picture [inset of Fig. 1(a)], which shows a similar pattern, however, with reduced statistics.

It is possible to investigate the mean lateral aspect ratio of the elongated QDs and its impact on the strain field in a more accurate way. In a previous investigation on elongated $\mathrm{InP} / \mathrm{InGaP}$ quantum dots, scaling laws between the QD geometrical aspect ratios and the mean lateral strain relaxation have been deduced. ${ }^{7}$ These scaling laws are valid as long as the height of the QD is much smaller than the lateral QD base widths. In particular, the mean lateral strain inside the islands $\varepsilon$ scales inversely with the base width $w$. In order to probe the lateral strain field inside and close to the QDs, grazing incidence $\mathrm{x}$-ray diffraction was performed in the proximity of the in-plane $0 \overline{2} 2$ and 022 reciprocal lattice points [Figs. 2(a) and 2(b), respectively]. The peak positions $F_{2}$ and $F_{1}$ are used to calculate the mean lateral total strain in the QDs as $\varepsilon_{011}=4.1 \times 10^{-3}$ and $\varepsilon_{011}=5.9 \times 10^{-3}$. These values are then used to evaluate the lateral geometrical aspect ratio of the dots as $\alpha=w_{011} / w_{011}=1.44$, which turns out to be in good agreement with GISAXS and AFM.

While the elongation of the dots $(\alpha=1.44)$ is not very pronounced, it leads to an anisotropic strain relief in the neighboring GaAs substrate. This anisotropy is proved by numerical calculations based on linear elasticity theory (Fig. 3 ). These calculations show distinct differences in the lateral o AlP license or copyright; see http://apl.aip.org/apl/copyright.jsp 


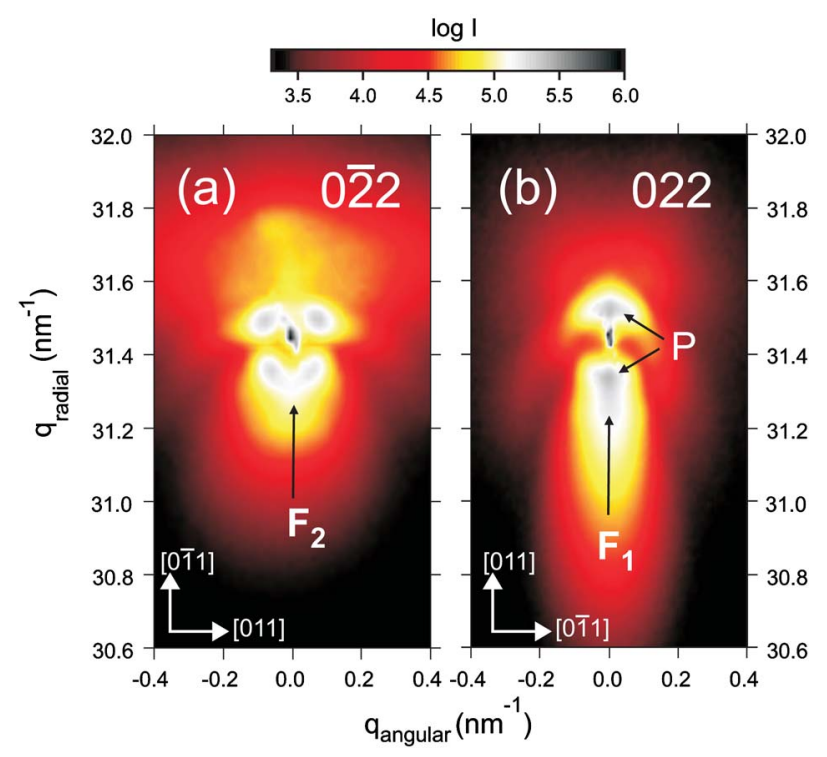

FIG. 2. (Color online) GIXD patterns of a single layer of $\operatorname{In}_{0.40} \mathrm{Ga}_{0.60}$ As QDs grown on $\operatorname{GaAs}(100)$ (sample 1) in the vicinity of the (a) $(0 \overline{2} 2)$ and (b) 022 reciprocal lattice point.

(total) strain components $\varepsilon_{x x}$ and $\varepsilon_{y y}$, where the $x$ and $y$ axes are collinear with the planar [011] and [011] directions, respectively. In the neighborhood of the island, the strain tensor components $\varepsilon_{x x}$ and $\varepsilon_{y y}$ exhibit different signs depending on the position around the elongated island, indicating tensile and compressive strain with respect to the GaAs substrate. More interesting is the spatial dependence of the strain fields as a function of the lateral distance to the island. For the tensile strained areas, we can state that $\varepsilon_{y y}^{+}$is always smaller than $\varepsilon_{x x}^{+}$, and the same statement holds for compressively strained areas $\left(\left|\varepsilon_{y y}^{-}\right|<\left|\varepsilon_{x x}^{-}\right|\right)$. In other words, $\varepsilon_{y y}$ decays on a shorter length scale as compared to $\varepsilon_{x x}$, indicating a pronounced anisotropic strain relaxation, which is induced by the asymmetric island shape.

So far we have discussed the growth scenario in terms of strain alone. However, we would like to stress that the evolution of QD chains is a result of nucleation and growth for the individual islands. Therefore, strain mediated adatom diffusion has to be taken into account. There is, up to now, only little knowledge about the impact of epitaxial strain onto the adatom diffusion tensor, i.e., onto the anisotropy of diffusion. Penev et al. ${ }^{18}$ found for the example of In/GaAs(001)-c(4 $\times 4)$ that the diffusion barriers are lowered by tensile stress. Anisotropic strain thus induces anisotropy in surface diffusion. When applying the results obtained by Penev et al. to our case of elongated (In,Ga)As islands, we can conclude that the anisotropic strain field in the vicinity of the islands (as presented in Fig. 3) further enhances the anisotropy in diffusion lengths along [011] and [0 $\overline{1} 1]$.

Based on the finite element calculations, we can therefore state that the elastic strain field in the vicinity of an elongated single island plays a key role in the formation of perfect QD chains in the multilayered sample. At growth conditions close to thermodynamical equilibrium, the anisotropy in the strain field leads to an anisotropic adatom nucleation and, eventually, to an asymmetric separation between neighboring dots in these two directions. Obviously, the elongated QD shape along [0 $\overline{1} 1]$ leads to preferential alignment of neighboring dots in these directions as indeed ob-

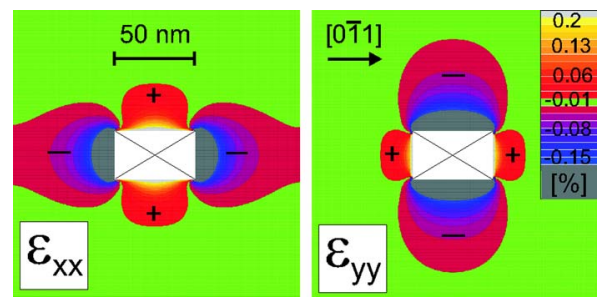

FIG. 3. (Color online) Numerical finite element calculations of the total strain tensor components $\varepsilon_{x x}$ and $\varepsilon_{y y}$ in the vicinity of an elongated $\mathrm{In}_{0.40} \mathrm{Ga}_{0.60} \mathrm{As}$ island grown on $\mathrm{GaAs}(100)$. The $\mathrm{In}_{0.40} \mathrm{Ga}_{0.60} \mathrm{As}$ wetting layer thickness has been assumed as $2 \mathrm{~nm}$.

served for sample 1 [Fig. 1(a)]. When growth of the first QD layer is completed and the QD layer is covered by a thin GaAs spacer layer, the strain field of the buried QDs is left as a fingerprint on the GaAs surface. When growth of the next $\mathrm{In}_{0.40} \mathrm{Ga}_{0.60}$ As layer starts, this strain field amplifies the "intrinsic" weak alignment caused by the elongated QD shape and serves as a template for the formation of QD chains. Therefore, the ordering of the dots is improved from layer to layer and after growth of many layers perfectly aligned QD chains may evolve. This mechanism of strain inheritance thus favors the evolution of monodisperse islands with very high lateral ordering as predicted by Tersoff et al. ${ }^{19}$

The authors appreciate technical support by beamline staff of ESRF and HASYLAB and financial support by the Deutsche Forschungsgemeinschaft in the framework of Sonderforschungsbereich 296 (project A3). One of the authors (M.H.) acknowledges financial support by the Federal State of Sachsen-Anhalt, Germany, within the Cluster of Excellence (CoE) on Nanostructured Materials (project NW3).

${ }^{1}$ D. Leonard, M. Krishnamurthy, C. M. Reaves, S. P. Denbaars, and P. M. Petroff, Appl. Phys. Lett. 63, 3203 (1993).

${ }^{2}$ T. Mano, R. Nötzel, G. J. Hamhuis, T. J. Eijkemans, and J. H. Wolter, Appl. Phys. Lett. 81, 1705 (2002).

${ }^{3}$ Z. M. Wang, K. Holmes, Yu. I. Mazur, and G. J. Salamo, Appl. Phys. Lett. 84, 1931 (2004).

${ }^{4}$ W. Q. Ma, M. L. Hussein, J. L. Shultz, G. J. Salamo, T. D. Mishima, and M. B. Johnson, Phys. Rev. B 69, 233312 (2004).

${ }^{5}$ M. Schmidbauer, Sh. Seydmohamadi, D. Grigoriev, Zh. M. Wang, Yu. I. Mazur, P. Schäfer, M. Hanke, R. Köhler, and G. J. Salamo, Phys. Rev. Lett. 96, 066108 (2006).

${ }^{6}$ B. L. Liang, Zh. M. Wang, Yu. I. Mazur, V. V. Strelchuck, K. Holmes, J. H. Lee, and G. J. Salamo, Nanotechnology 17, 2736 (2006).

${ }^{7}$ M. Schmidbauer, F. Hatami, M. Hanke, P. Schäfer, K. Braune, W. T. Masselink, R. Köhler, and M. Ramsteiner, Phys. Rev. B 65, 125320 (2002).

${ }^{8}$ M. Sugisaki, H. Ren, S. V. Nair, K. Nishi, S. Sugou, T. Okuno, and Y. Masumoto, Phys. Rev. B 59, R5300 (1999).

${ }^{9}$ G. Medeiros-Ribeiro, A. M. Bratkowski, T. I. Kamins, D. A. A. Ohlberg, and R. S. Williams, Science 279, 353 (1998).

${ }^{10}$ I. Kegel, T. H. Metzger, A. Lorke, J. Peisl, J. Stangl, G. Bauer, J. M. Garcia, and P. M. Petroff, Phys. Rev. Lett. 85, 1694 (2000).

${ }^{11}$ Ch. Heyn, Phys. Rev. B 63, 033403 (2001).

${ }^{12}$ E. Pehlke, N. Moll, A. Kley, and M. Scheffler, Appl. Phys. A: Mater. Sci. Process. 65, 525 (1997).

${ }^{13}$ J. Tersoff and R. M. Tromp, Phys. Rev. Lett. 70, 2782 (1993).

${ }^{14}$ Y. W. Mo, D. E. Savage, B. S. Swartzentruber, and M. G. Lagally, Phys. Rev. Lett. 65, 1020 (1990).

${ }^{15}$ P. Ballett, J. B. Smathers, H. Yang, C. L. Workman, and G. J. Salamo, Appl. Phys. Lett. 77, 3406 (2000).

${ }^{16}$ J. Marquez, L. Geelhaar, and K. Jacobi, Appl. Phys. Lett. 78, 2309 (2001).

${ }^{17}$ A. Lorke, R. J. Luyken, A. O. Govorov, J. P. Kotthaus, J. M. Garcia, and P. M. Petroff, Phys. Rev. Lett. 84, 2223 (2000).

${ }^{18}$ E. Penev, P. Kratzer, and M. Scheffler, Phys. Rev. B 64, 085401 (2001).

${ }^{19}$ J. Tersoff, C. Teichert, and M. G. Lagally, Phys. Rev. Lett. 76, 1675 (1996). 